foot and lameness. On admission to hospital the left knee was flexed and conld not be straightened, and movement of it occasioned some pain. There was a swelling as big as a duck's egg occupying the site of the head of the fibula and extending into the popliteal space. The patient had lost flesh lately, looked more than his age, had had recent trouble (baving lost his wife), and wandered a little at night. The case was diagnosed by me as sarcoma of the ham, and amputation at the thigh was recommended should an exploratory incision into the tumour confirm the diagnosis. On April 3rd amputation at a little below the middle of the thigh was performed under chloroform. He bore the operation very well. For a week following the operation the temperature fluctuated between $98^{\circ}$ and $103^{\circ} \mathrm{F}$. The delirium at night was more marked. On the 11th he had intractable diarrhoea, was delirious, and was losing ground. On the 13th be was passing arine and motions involuntarily. Ether (6 drachms in the twenty-four hours) was ordered. On the 14th the temperature was running higher; there was continuous involuntary diarrhoea unchecked by opium, and he was busily delirious and sleeping badjy. The pulse was very rapid -140 and more. Spiritus vini rectilicatus was ordered, one drachm to be given every four hours with egg and milk unless he was drowsy. On the 15th he had no sleep; he still took food fairly well. There was no bed-sore. The alcohol was continued every six hours. On the 16th he died.

Post mortem secondary deposits of sarcomatous nature were found in the liver; a large cavity containing new growth occupied the iliac region; there was also an abscess in the right inguinal region. There was scarcely any tendency towards repair in the flaps, but there was no osteomyelitis or tracking of pus. The other organs were healthy.

In two of the above cases only was alcobol in any form administered, the case just related being one of the two. The other case was one of amputation of the leg for embolic gangrene in a flabby woman aged forty-nine years with cardiac disease, who recovered. Full details of this and of all other cases in which alcohol is administered are set out in the annual reports of the hospital in accordance with Rule XV. of its constitution, which provides that: "It shall be incumbent on the prescribing medical officer, in any case where alcohol in any form is ordered for a patient, to enter in a book kept for that purpose the full particulars of the prescription, inclading the time, quantity, and reasons of administration. The effect of the dose also shall subsequently be noted."

Albert-terrace, Regent's-park, N.W.

\section{SCHOOL-MADE INSANITY.}

\section{BY LL. HARRIS-LISTON, M.R.C.S. ENG., L.R.C.P. LOND.,} L.S.A.,

ASSISTAYT IIEDICAL OFFICER, HOSPITAL FOR MENTAL DISEASES, COION BILL, STATFORD.

THE classical statement made by the old-time surgeon, that no injury to the head which produces insensibility is too trivial to be disregarded or too serious to be despaired of, is one the truth of which is frequently in evidence. A case which has recently been under my observation may be guoted as an example, and for other reasons is, I think, worthy of record. It is a case in which a few blows given with the open hand on the side of the head by one boy to another was the exciting cause of attacks of insanity of some years' duration. Although no cause for an attack of insanity is more frequently assigned by parents than a fall or blow on the head during infancy, on investigation some zore probable factor is generally to be found. In the report of the Lunacy Commissioners for 18963 per cent. only is the proportion of cases in which injury is given as the predisposing or exciting cause in all cases admitted into asplums, hospitals, or licensed houses during the five years 1890-94. In this case the hereditary history is remarkably clear of all nervous diseases, the only instance being an attank of senile dementia in the patient's paternal grandfather at an advanced age.

The first attack began when the patient, then sixteen years of age, was at school, the symptoms being mental depression, sleeplessness, pain in the head, unsociable habits, and loss of nutrition coming on within two months of the injuries. He was taken from school, and after spending a few months at home recovered his natural spirits, became a clerk, and remained well for two years. Then, at eighteen years of age, he had an attack of acute mania, for which be was treated at a lunatic hospital and from which he recovered in eight months and resumed his work as a clerk. For six years he remained well, spending the last year of this period in Canada farming. Then, becoming depressed, fearing that he was about to have another attack, and feeling self-conscious amongst strangers, he lost all interest in his work and, without giving his relatives any warning, suddenly arrived at home. $\mathrm{He}$ gradually got worse in mind and body, became despondent, silent, morose, lost all energy, avoided society, refused food, and became emaciated and weak; therefore, he was certified again and admitted, at the age of twenty-five years, to a lunatic hospital, where he became more confused and obstinate and in a few months sank into a state of stupor. After twelve months' residence in this hospital, as he became no better, a change of environment was advised and he was transferred to another asylum, where I first saw him. He was then in a state of melancholic stupor, being resistive and conscious, with a miserable, furtive expression, standing in one place and in the same attitude for hours, with a band pressed to the head or stomach, taking food unwillingly and sparingly, answering no questions unless repeatedly urged, and then only giving a monosyllabic reply. He was unsettled, restless and sleepless at night, thin, with flabby muscles. The pulse was 64 and weak, the temperature $97^{\circ} \mathrm{F}$., the bowels constipated, the extremities cold, and the circulation poor. He remained in this state for two years longer, the state of stupor having lasted altogether for three years, then be gradually began to take some interest in his surroundings and answer simple questions in a whisper. He was then induced to do some outdoor work, and in three months gained flesh and some confidence, but still thinking badly of himself and of his bodily condition. Questioned as to his past state of stupor be said that he remembered everytbing that had happened around him and that he had of ten crept away to avoid observation when strangers visited the hospital, that be had feelings altogether different from, and bodily sensations sirange to, those he had when in health. In a few months more be still further improved, getting strong in body, slightly hypochondriacal, but having some hope of regaining a position in the outside world. He was discharged as recovered. The treatment of his last attack consisted of giving quinine and strychnine in large doses, malt extract, plenty of food and alcoholic stimulants, warm baths, cold douches, and latterly of manual work out of doors, bicycling, golf, swimming, cricket, \&c.

The causation of this case is its interesting feature : blows on the head with the open band severe enough to cause unconsciousness on at least two occasions, without a lesion of the bone, but producing some molecular disturbance of the brain sufficient to excite insanity. The injury was inflicted as punishment for some minor offences by one of a body of boy prefects, who at the school the patient attended were allowed disciplinary powers, one being to strike a boy on the head, apparently as often and as hard as they deemed advisable; surely a dangerous power to entrust to boys. There are other contributory conditions in the case only prevailing at this period of life, and which exist at many other schools, and which have caused me to call the case "schoolmade": the age of puberty, one of the periods of life at which nervous diseases are prone to appear; the reprehensible practice of working scholars in the early morning before breakfast and after a long fast; and the emotional influence of constantly recurring religious services carried out in some schools to a ridiculous extent.

Stafford.

The secretary of the East London Hospital for Children calls attention to the need of funds for current maintenance, and of from $£ 1000$ to $£ 1500$ to complete the estimated cost of the Convalescent Home which the managers are about to erect at Bognor.

THe total of the subscriptions for the Memorial erected over the grave of the late Mr. Reese of Ystradgynlais, who was killed by lightning on July 21st, 1895, has reached the sum of $£ 1020 s$. $11 d$. 